\title{
Uso e interpretação das razões de concentração e sua aplicação à análise da insegurança alimentar no Brasil
}

\author{
Nova versão de abril de 2017, com correção das Figuras 2 e 3
}

\section{Rodolfo Hoffmann ${ }^{1}$}

O uso crescente das curvas de concentração e das razões de concentração em pesquisas sobre problemas de saúde pública torna oportuno analisar cuidadosamente esses instrumentos de análise estatística. Após uma breve introdução, a seção principal deste artigo procura apresentar com clareza as curvas de concentração e as respectivas razões de concentração, mostrando que elas não são, em geral, medidas de desigualdade de uma distribuição. São criticadas duas propostas de "corrigir" as razões de concentração. Nas seções seguintes esses instrumentos são utilizados para analisar a concentração da insegurança alimentar no Brasil, em relação à renda, com dados de 2004, 2009 e 2013. Não se observa mudança substancial do grau dessa concentração no período analisado. Mostra-se que a concentração da insegurança alimentar nos pobres é mais intensa na área urbana do que na área rural. Analisando os dados por região do país, verifica-se que essa concentração é mais intensa nas regiões relativamente ricas do Centro-Sul do que nas duas regiões mais pobres: Norte e Nordeste.

Palavras-chave: Razões de concentração; curvas de concentração; segurança alimentar.

\section{Use and interpretation of concentration ratios and their application to the analysis of food insecurity in Brazil}

The increasing use of concentration curves and concentration ratios in public health studies demands a careful analysis of these statistical tools. After a brief introduction, the main section of this paper explains the meaning of the concentration curves and the associated concentration ratios, stressing that they are not, in general, measures of the inequality of a distribution. Two proposals for a supposed correction of the concentration ratios are criticized. In the subsequent sections these statistical tools are used to analyze the concentration of food insecurity in Brazil, in relation to income, in 2004, 2009 and 2013. The results show that this concentration is rather stable in the period. The concentration of food insecurity in the poorest groups of the population is more intense in the urban than in the rural area and is more intense in the center-south regions than in the poorer North and Northeast regions.

Keywords: Concentration ratios; concentration curves; food security.

${ }^{1}$ Professor Sênior da Escola Superior de Agricultura “Luiz de Queiroz” (ESALQ), da Universidade de São Paulo (USP), com apoio do CNPq. E-mail: hoffmannr@usp.br 


\section{INTRODUÇÃO}

Cresce o uso das curvas de concentração e das razões de concentração em estudos de saúde pública. Para isso contribui o livro bastante didático de O’Donnell et al. intitulado "Analysing health equity using household survey data: a guide to techniques and their implementation" [1]. É conveniente discutir melhor os conceitos subjacentes, deixando claro que uma razão de concentração nula não significa que a variável analisada seja igualitariamente distribuída na amostra.

Na próxima seção são apresentadas as definições de curva de concentração e razão de concentração, procurando deixar clara a interpretação desses instrumentos estatísticos. Posteriormente eles serão utilizados para analisar os dados sobre insegurança alimentar no Brasil em 2004, 2009 e 2013.

\section{Razões de concentração e curvas de concentração}

É interessante iniciar a apresentação recordando o conceito do índice de Gini, que é a mais usual das medidas de desigualdade.

Se $X_{i}(\operatorname{com} i=1,2, \ldots, n)$ é o valor de uma variável em uma população com $n$ unidades (comumente $X_{i}$ é a renda da $i$-ésima pessoa), o seu valor médio é,

$$
\mu_{X}=\frac{1}{n} \sum_{i=1}^{n} X_{i}
$$

A diferença média (média do valor absoluto das diferenças entre dois valores quaisquer) é

$$
\Delta_{X}=\frac{1}{n^{2}} \sum_{i=1}^{n} \sum_{j=1}^{n}\left|X_{i}-X_{j}\right|
$$

e o índice de Gini pode ser definido como

$$
G_{X}=\frac{\Delta_{X}}{2 \mu_{X}}
$$

Vamos admitir que os valores de $X_{i}$ estejam ordenados de maneira que $X_{1} \leq X_{2} \leq X_{3} \leq \cdots \leq X_{n}$. Então $i$ indica a posição de ordem de cada um dos valores colocados em ordem crescente. Essa ordenação é fundamental para uma outra maneira de calcular o índice de Gini, que é

$$
G_{X}=\frac{2}{n \mu_{X}} \operatorname{cov}\left(i, X_{i}\right)
$$

Essa expressão mostra que o índice de Gini cresce com a covariância entre $X_{i}$ e sua própria posição de ordem. Havendo variação em $X_{i}$, essa covariância é necessariamente positiva.

Pode-se demonstrar que, para um conjunto de $n$ observações de $X_{i}$, têm-se

$$
0 \leq G_{X} \leq 1-\frac{1}{n}
$$

A proporção acumulada das unidades (população) até certo ponto é dada por

$$
p_{i}=\frac{i}{n}
$$

e a respectiva proporção acumulada dos valores $X_{i}$ é

$$
\Phi_{i}(X)=\frac{1}{n \mu_{X}} \sum_{j=1}^{i} X_{j}
$$

A curva que mostra como $\Phi_{i}(X)$ varia em função de $p_{i}$ é denominada curva de Lorenz. A Figura 1 mostra a curva de Lorenz (linha vermelha) da distribuição da renda domiciliar per capita (RDPC) no Brasil, de acordo com os dados da Pesquisa Nacional por Amostra de Domicílios (PNAD) de 20132. O segmento do bissetor do primeiro quadrante que vai da origem do sistema de eixos ao ponto $(1 ; 1)$ (a linha azul) é denominado linha da perfeita igualdade e a área entre essa linha e a curva de Lorenz é a área de desigualdade $\left(\alpha_{X}\right)$. Curiosamente, o dobro da área de desigualdade é exatamente o mesmo índice de Gini dado por (3) ou (4), isto é,

$$
G_{X}=2 \alpha_{X}
$$

Sendo $\beta_{X}$ a área entre a curva de Lorenz e o eixo das abscissas, verifica-se que $\alpha_{X}=0,5-\beta_{X}$. Substituindo em (8), segue-se que

$$
G_{X}=1-2 \beta_{X}
$$

\footnotetext{
2 São considerados todos os domicilios particulares permanentes com informação da
renda domiciliar (inclusive os com renda declarada igual a zero), excluindo as pessoas cuja

2 São considerados todos os domicílios particulares permanentes com informação da
renda domiciliar (inclusive os com renda declarada igual a zero), excluindo as pessoas cuja condição na unidade domiciliar é pensionista, empregado doméstico ou parente de empregado doméstico.
} 
Figura 1. Curva de Lorenz da distribuição da RDPC no Brasil de acordo com dados da PNAD de 2013

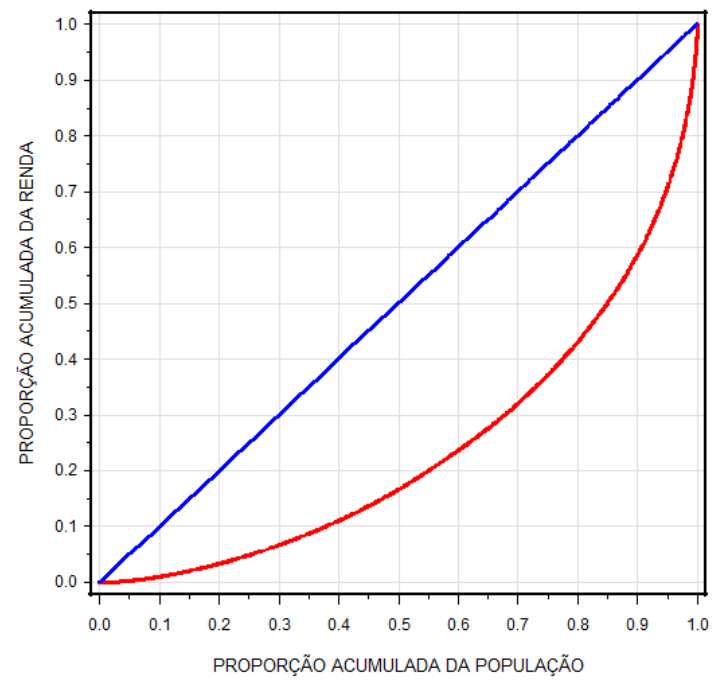

Fonte: Elaborada a partir dos microdados da PNAD de 2013

Observa-se, na Figura 1, que os $40 \%$ mais pobres da população ficam com pouco mais de $10 \%$ da renda total e os $80 \%$ com menores valores da RDPC recebem aproximadamente $43 \%$ da renda total, de maneira que os $20 \%$ mais ricos ficam com cerca de $57 \%$ da renda total.

Outra maneira de obter o índice de Gini é a expressão

$$
G=\frac{2}{n^{2} \mu_{X}} \sum_{i=1}^{n} i X_{i}-\left(1+\frac{1}{n}\right)
$$

É, realmente, algo curioso o número de diferentes fórmulas encontradas na literatura para calcular o mesmíssimo índice.

Vamos continuar admitindo que as $n$ observações da população estão ordenadas conforme valores crescentes de $X_{i}$. Consideremos, agora, uma outra variável $Y_{i}$. Para exemplificar, $X_{i}$ pode ser a RDPC e $Y_{i}$ pode ser o valor per capita de um imposto, ou o valor per capita da renda obtida de aluguéis, ou o índice de massa corporal da pessoa ou, ainda, uma variável binária indicando se o domicílio tem ou não insegurança alimentar grave.
Sendo $\mu_{Y}$ a média de $Y_{i}$ na população, a razão de concentração ${ }^{3}$ de $Y$ em relação a $X$ pode ser definida como

$$
C_{Y}=\frac{2}{n \mu_{Y}} \operatorname{cov}\left(i, Y_{i}\right)
$$

É importante lembrar que $i$ indica a posição de ordem de um par $\left(X_{i}, Y_{i}\right)$ com as observações ordenadas conforme valores crescentes de $X_{i}$ (que será, em geral, diferente da ordenação conforme valores de $Y_{i}$ ).

Comparando (4) e (11) verifica-se que o índice de Gini é a razão de concentração de $X_{i}$ em relação ao próprio $X_{i}$.

De (11) pode-se deduzir que ${ }^{4}$

$$
C_{Y}=\frac{2}{n^{2} \mu_{Y}} \sum_{i=1}^{n} i Y_{i}-\left(1+\frac{1}{n}\right)
$$

\footnotetext{
3 Também denominado indice de concentração. Não confundir com a "razão de concentração" usada para medir o grau de concentração de uma indústria, que é, simplesmente, a participação das $k$ maiores empresas (com $k=1,2, \ldots)$ em alguma medida de tamanho dessa indústria (ou ramo de atividades).

${ }^{4}$ Em Kakwani et al., 1997 [2] é apresentada uma fórmula ligeiramente diferente (falta subtrair $1 / n)$.
} 
Respeitando, sempre, a ordenação das observações conforme valores crescentes de $X_{i}$, a proporção acumulada dos valores de $Y_{i}$ até a $i$-ésima observação é

$$
\Phi_{i}(Y)=\frac{1}{n \mu_{Y}} \sum_{j=1}^{i} Y_{j}
$$

A curva mostrando como $\Phi_{i}(Y)$ varia em função de $p_{i}=i / n$ é denominada curva de concentração de $Y$ em relação a $X$.

Seja $\beta_{Y}$ a área entre a curva de concentração de $Y$ em relação a $X$ e o eixo das abcissas. Pode-se demonstrar que o valor da razão de concentração dado por (11) é idêntico a

$$
C_{Y}=1-2 \beta_{Y}
$$

Note-se a analogia entre (9) e (14).

Pode-se demonstrar que para um conjunto de $n$ observações $\left(X_{i}, Y_{i}\right)$,

$$
-1+\frac{1}{n} \leq C_{Y} \leq 1-\frac{1}{n}
$$

Se $X$ é a renda das pessoas, um valor elevado (próximo de 1) de $C_{Y}$ indica que $Y$ está concentrado nos relativamente ricos e um valor baixo (próximo de -1) indica que $Y$ está concentrado nos pobres.

Se $Y$ for igualitariamente distribuída na população, isto é, se $Y_{i}=\mu_{Y}$ para todo $i$, a covariância entre $i$ e $Y_{i}$ será nula e, conforme (12), a razão de concentração de $Y$ em relação a $X$ será igual a zero $\left(C_{Y}=0\right)$.

Mas o inverso não é verdadeiro. Se $C_{Y}=0$ não se pode concluir que a distribuição de $Y$ é igualitária. A covariância entre $Y_{i}$ e $i$ pode ser nula mesmo havendo grande desigualdade na distribuição de $Y$. O fato de termos $Y_{i}=\mu_{Y}$ para todo $i$ é condição suficiente, mas não necessária, para $C_{Y}=0$.

Há erros, portanto, na afirmativa de que "o índice de concentração é semelhante ao coeficiente de Gini - varia de $-1 \mathrm{a}+1$ e o valor 0 indica igualdade total na distribuição do desfecho" (Monteiro et al., 2013 [3], p. 37).
A ideia errônea de que a razão de concentração mede "desigualdade" pode ser encontrada em outros artigos como, por exemplo, Wagstaff (2005 [4], p. 431).

O mesmo autor, em artigo de 1991 [5], denomina a razão de concentração de medida de desigualdade da saúde, certamente considerando implícito que se trata da desigualdade de saúde associada à desigualdade em status socioeconômico.

Para medir a desigualdade da distribuição de $Y$ deve-se calcular o índice de Gini da distribuição de $Y$ (ou outra medida apropriada de desigualdade).

Nem mesmo o fato de a curva de concentração se confundir com o bissetor do primeiro quadrante (uma condição suficiente, mas não necessária, para termos $C_{Y}=0$ ) garante que $Y$ tenha distribuição igualitária. Considere-se, por exemplo, uma amostra com $n=1001$ observações na qual aos valores $i=\{1,2,3,4, \ldots, 1000$, $1001\}$, referentes à ordenação $\operatorname{dos} X_{i}$, correspondem os valores $Y_{i}=\{0,1,0,1, \ldots, 1,0\}$. Verifica-se que $\operatorname{cov}\left(i, Y_{i}\right)=0, C_{Y}=0$ e em um gráfico com a curva de concentração em uma folha de papel comum essa curva se confunde com o bissetor do primeiro quadrante. Entretanto, a distribuição de $Y$ não é igualitária e o respectivo índice de Gini é praticamente igual a 0,5 . Em um gráfico da curva de Lorenz, é apropriado denominar o bissetor do primeiro quadrante de "linha da perfeita igualdade". Mas essa denominação deixa de ser apropriada quando se trata de curvas de concentração em geral.

A Figura 2 mostra a curva de Lorenz (linha preta) da distribuição da RDPC e 4 curvas de concentração obtidas a partir dos microdados das PNAD de 2013. A curva referente ao rendimento de aluguéis (valor per capita em cada domicilio, que é uma parcela da RDPC) é a mais concentrada. A respectiva razão de concentração é igual a 0,790 , maior do que o índice de Gini da RDPC, que é 0,525 . Note-se que rendimentos de aluguéis são praticamente inexistentes para o quinto mais pobre da população, fazendo com que nesse intervalo a curva de concentração fique praticamente sobre o eixo das abcissas.

A curva referente ao valor domiciliar per capita do rendimento recebido na forma de salário de trabalhadores agrícolas temporários sem carteira de trabalho assinada fica, em geral, acima do bissetor do primeiro quadrante e a respectiva razão de concentração é negativa: $-0,287$. A curva sobe rapidamente na cauda esquerda da distribuição da RDPC, pois esse tipo de renda é mais importante para domicilios pobres, como mostra a Figura 3. Para o décimo mais pobre da 
população brasileira esse tipo de rendimento representa quase $4 \%$ do total.

Figura 2. Curva de Lorenz e curvas de concentração para rendimento de empregados agrícolas temporários sem carteira, rendimento de empregados domésticos, rendimento de aluguéis e uma variável binária para posse de carro, de acordo com dados da PNAD de 2013

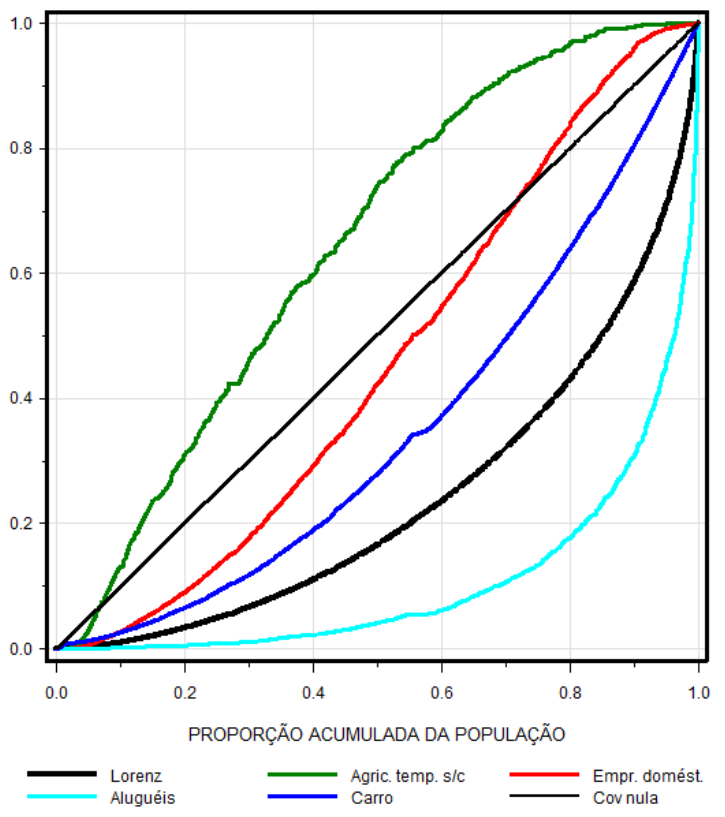

Figura 3. Participação do rendimento de empregados agrícolas temporários sem carteira na renda domiciliar em cada décimo da distribuição da RDPC

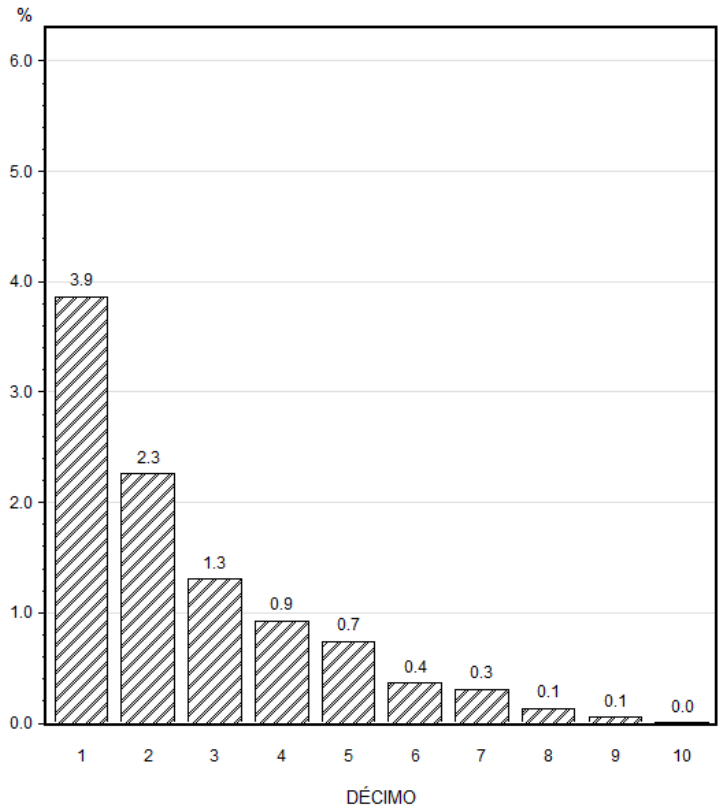


A curva de concentração do rendimento do trabalho de empregados domésticos cruza o bissetor do primeiro quadrante um pouco acima do sétimo decil da distribuição da RDPC. A área sob essa curva é aproximadamente igual a 0,5 (que é a área do triângulo abaixo do bissetor), de maneira que a respectiva razão de concentração é baixa (igual a 0,093).

Consideremos, finalmente, a curva de concentração de uma variável binária que indica, para cada pessoa, se o respectivo domicilio tem (valor 1 ) ou não tem (valor 0) carro de uso pessoal. Como mostra a Figura 4, a proporção de pessoas cujos domicílios tem carro é $11,3 \%$ no décimo mais pobre e alcança $87,2 \%$ no décimo mais rico da distribuição da RDPC. A proporção na população toda é $44,5 \%$. Portanto a curva de concentração cresce mais lentamente para os relativamente pobres e mais rapidamente para os relativamente ricos, como mostra a Figura 2. A respectiva razão de concentração é 0,306 , positiva, mas menor do que o índice de Gini. É claro que o resultado seria diferente se fosse considerado o valor dos carros em cada domicílio, e não uma variável binária indicando se há ou não carro no domicílio. É provável que a razão de concentração do valor dos carros seja até maior do que o índice de Gini da distribuição da RDPC. Como 55,5\% das pessoas estão em domicílios que não têm carro, o índice de Gini da distribuição da variável binária é igual a 0,555 . Para uma variável binária com valores 0 e $\theta>0$ o índice de Gini é igual à proporção de valores nulos.

Figura 4. Proporção de pessoas em domicílios com carro em cada décimo da distribuição da RDPC

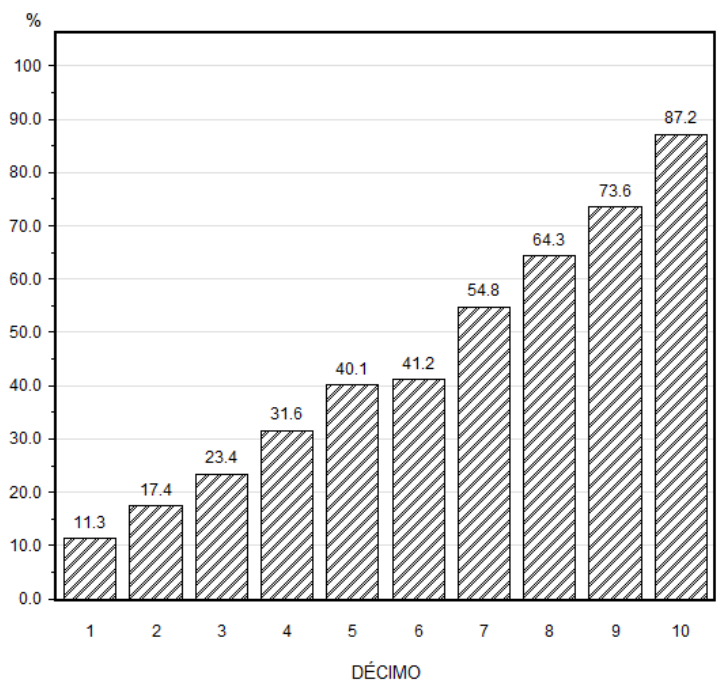

Como ilustração adicional do uso de razões de concentração para variáveis binárias, o Apêndice A mostra a evolução, de 1995 a 2013, das razões de concentração da posse de vários bens de consumo duráveis em relação à RDPC, no Brasil.

Curvas de concentração obtidas com dados da Pesquisa de Orçamentos Familiares (POF) de 2008-2009, incluindo a referente às transferências do Programa Bolsa Família, são apresentadas em Hoffmann, 2013 [ []. A razão de concentração das transferências do Programa Bolsa Família em relação à renda familiar per capita é igual a $-0,562$, mostrando que os benefícios do programa estão razoavelmente bem focalizados nos pobres.

\section{Uma "correção" inapropriada}

Consideremos uma variável $Y$ binária, com valores 0 e 1 . Então a sua média $\left(\mu_{Y}\right)$ é igual à proporção de valores iguais a 1 . Wagstaff (2005) [4], ressaltou que nesse caso a razão de concentração $C_{Y}$ é limitada por ${ }^{5}$

$$
\mu_{Y}-1 \leq C_{Y} \leq 1-\mu_{Y}
$$


Ele ressalta que o intervalo de possível variação de $C_{Y}$ se torna muito estreito quando $\mu_{Y}$ é elevado (próximo de 1). Sugere, então, que seja considerado o valor de $C_{Y}$ como fração do limite, isto é, seja considerado o valor de $C_{Y} /\left(1-\mu_{Y}\right)$.

A sugestão de Wagstaff é inapropriada. Ele ilustra o que lhe parece um problema com dados sobre imunização em 41 países em desenvolvimento. Se a grande maioria da população já for imunizada, obviamente esse indicador não pode estar muito concentrado a favor dos ricos. No limite, se $100 \%$ da população estiver imunizada, não será possível captar qualquer concentração do indicador a favor dos ricos. Os limites estabelecidos na expressão (16) refletem, adequadamente, características do conceito de desigualdade e concentração. Não há razão pra "corrigir" o valor de $C_{Y}$.

É interessante analisar a questão na situação mais simples em que se deseja avaliar o grau de desigualdade da distribuição de uma variável binária $(Z)$ com valores 0 e 1 . A média da variável $\left(\mu_{Z}\right)$ é igual à proporção de valores iguais a 1 . O índice de Gini é igual à proporção de valores iguais a $0: G_{Z}=1-\mu_{Z}$ para $\mu_{Z}>$ 0 ( $G_{Z}$ não é definido se $Z$ for sempre igual a 0$)$. A desigualdade, como medida pelo índice de Gini, é máxima quando $Z=1$ para uma única observação. Seria obviamente absurdo "corrigir" o índice de Gini nesse caso dividindo-o por $1-\mu_{Z}$.

Em 2009 Erreygers [7] apresenta e defende uma nova "correção" da razão de concentração. $\mathrm{O}$ autor parte de um conjunto de axiomas incompatível com a noção usual de desigualdade (e concentração). O máximo de desigualdade em uma distribuição de renda (para valores não negativos) ocorre quando uma única pessoa fica com toda a renda. Analogamente, é coerente considerar que a concentração de um benefício qualquer em relação à renda é máxima quando o único beneficiado é o mais rico da população. Mas a nova medida de concentração de Erreygers atinge valor máximo quando a metade relativamente rica da população recebe o benefício (boa saúde, no seu exemplo) e a outra metade não. Apesar de Erreygers denomina-la "uma nova medida de desigualdade", ela não pode ser usada como tal, pois o seu valor não é afetado quando se soma uma constante a todos os valores (o que levaria a dizer que a desigualdade entre 1, 3 e 20 é igual à desigualdade entre 1001, 1003 e $1020)^{6}$.

Um dos axiomas adotados por Erreygers [7] estabelece que a medida de concentração de uma variável

${ }^{6}$ A nova medida de Erreygers [7] é criticada por Wagstaff ${ }^{[8]}$ e há réplica de Erreygers ${ }^{[9]}$. (saúde) em relação a $X$ deve ter valor absoluto igual e sinal contrário à medida de concentração da variável complementar (falta de saúde) em relação a $X$. Assim, se a medida de concentração de uma variável binária $Y$, com valores 0 e 1 , em relação a $X$ for igual a $\beta$, a medida de concentração de $Z=1-Y$ em relação a $X$ deve ser igual a $-\beta$. Parece razoável, mas não é.

Imagine uma comunidade com 100 pessoas onde apenas o mais rico tem carro. Seja $Y$ a variável binária que é igual a 1 quando a pessoa tem carro. A média dessa variável é $\mu_{Y}=0,01$ e o respectivo índice de Gini é $G_{Y}=0,99$. A razão de concentração de $Y$ em relação à renda $\left(C_{Y}\right)$ também é igual a 0,99 .

Imaginemos, agora, que a pessoa mais rica dessa comunidade resolve viver livre de todos os bens materiais, usando toda sua fortuna para dar um carro para as outras 99 pessoas da comunidade. Assim ele se torna o mais pobre e o único sem carro. Nessa nova situação temos $\mu_{Y}=0,99, G_{Y}=0,01$ e a razão de concentração de $Y$ em relação à renda é $C_{Y}=0,01$.

O índice de concentração "corrigido" de Erreygers [7] é igual a 0,04 (ou, exatamente, 0,0396) nos dois casos. Uma medida "corrigida" que perdeu a capacidade de distinguir duas situações muito distintas no que se refere à posse de carros em relação à renda.

Em minha opinião a nova medida de Erreygers [7] só causa confusão e deveria ser esquecida. Infelizmente Monteiro et al. ${ }^{[3]}$ levam a proposta dele a sério.

Imagine que algum indicador de saúde mostre situação "saudável" $(Y=1)$ apenas para o centésimo mais rico da população brasileira. Todos os demais estão em situação "não saudável" $(Y=0)$. Nessa situação hipotética a saúde está altamente concentrada nos ricos. A razão de concentração de saúde em relação à renda é $C_{Y}=0,99$, mas o índice de concentração de Erreygers é igual a 0,04 (exatamente 0,0396$)^{7}$. Consideremos, agora, a variável indicadora de falta de saúde $Z=1-Y$. Então $Z=1$ para os primeiros 99 centésimos da distribuição de renda. Apenas para o centésimo mais rico temos $Z=$ 0 . Não é razoável, nessa situação hipotética, pretender que a "falta de saúde" $(Z=1)$ está concentrada nos pobres da mesma maneira que a "saúde" $Y=1$ está concentrada nos ricos, já que a falta de saúde está disseminada por quase toda a população. A razão de concentração da "falta de saúde" $(Z)$ é $C_{Z}=-0,01$,

\footnotetext{
$7 \mathrm{O}$ índice de Erreygers para concentração da saúde em relação à renda atinge seu valor máximo (igual a 1) quando todas as pessoas com renda acima da mediana forem "saudáveis" e as demais forem "não saudáveis".
} 
mas o índice de Erreygers para falta de saúde tem valor absoluto igual ao do índice para saúde: é igual a $-0,04$.

Sempre que $Y$ for uma variável binária com valores 0 e 1 e $Z$ for seu complemento $(Z=1-Y)$, vale a seguinte relação entre suas razões de concentração:

$$
C_{Z}=-\frac{\mu_{Y}}{1-\mu_{Y}} C_{Y}
$$

onde $\mu_{Y}$ é a média de $Y$, que é a proporção da população $\operatorname{com} Y=1$.

\section{Análise das razões de concentração da insegurança alimentar no Brasil em 2004, 2009 e 2013}

Nos anos de 2004, 2009 e 2013 o IBGE incluiu, na PNAD, uma pesquisa suplementar sobre segurança alimentar, aplicando a Escala Brasileira de Insegurança Alimentar (EBIA) em cada domicílio da amostra. A descrição da escala e dos resultados básicos obtidos nos três levantamentos pode ser encontrada nas publicações do próprio IBGE (2006 [10], 2010 [11] e 2014 [12]). Atualmente a escala é baseada nas respostas a 14 perguntas e, conforme o número de respostas "sim" e a presença ou não de pessoa menor de 18 anos o domicílio é classificado em uma de 4 categorias: com segurança alimentar, com insegurança leve, com insegurança moderada ou com insegurança alimentar grave.

Vamos usar as curvas de concentração e as razões de concentração para avaliar o grau em que a segurança alimentar está concentrada nos pobres, isto é, na cauda esquerda da distribuição da RDPC. A RDPC é calculada dividindo, para cada domicílio, a renda domiciliar pelo número de pessoas do domicílio, exclusive os pensionistas e os empregados domésticos (e seus parentes) residentes no domicílio do empregador. A análise será restrita aos domicílios particulares permanentes com informação sobre a RDPC e a classificação em uma das 4 categorias de segurança ou insegurança alimentar8.
A unidade de análise é a pessoa. Dessa maneira, os dados de cada domicílio da amostra são ponderados por um fator que é igual ao produto do fator de expansão do domicílio (fornecido pelo IBGE) pelo número de pessoas do domicílio usado no cálculo da RDPC.

A Tabela 1 mostra o tamanho da população analisada e a proporção de pessoas em cada uma das quatro categorias de segurança alimentar em 2004, 2009 e 2013. Cabe ressaltar que no caso de 2004 não foi utilizada a variável-síntese de segurança alimentar disponível no arquivo de microdados (V4623 ou V4744). Conforme orientação do próprio IBGE, a classificação foi refeita seguindo critérios usados em 2009 e 2013 (ver Apêndice A em Hoffmann, 2014 [13]). 
Tabela 1. Pessoas em domicílios particulares permanentes com informação sobre RDPC e segurança alimentar e porcentagens de pessoas conforme categoria de segurança alimentar. Brasil: 2004, 2009 e 2013

\begin{tabular}{lrrr}
\hline \multicolumn{1}{c}{ Estatística } & $\mathbf{2 0 0 4}$ & $\mathbf{2 0 0 9}$ & $\mathbf{2 0 1 3}$ \\
\hline População (1000 pessoas) & 178.133 & 186.924 & 189.853 \\
\% com segurança alimentar & 59,9 & 65,5 & 73,8 \\
\% com insegurança leve & 20,4 & 21,0 & 17,4 \\
\% com insegurança moderada & 11,4 & 7,5 & 5,2 \\
\% com insegurança grave & 8,3 & 5,9 & 3,6 \\
\hline Fonte: Elaborada a partir dos microdados da PNAD (IBGE) & & &
\end{tabular}

Fonte: Elaborada a partir dos microdados da PNAD (IBGE)

As pequenas diferenças dos valores apresentados na Tabela $1 \mathrm{em}$ relação aos apresentados em IBGE, 2014 [12], p. 36, se devem ao fato de lá serem considerados todos os moradores de domicílios particulares. Excetuando-se o pequeno aumento da proporção de pessoas com insegurança leve de 2004 para 2009, que pode ser atribuído ao caráter parcialmente subjetivo da EBIA, principalmente para captar insegurança alimentar leve, há substancial crescimento da segurança alimentar e redução dos três níveis de insegurança alimentar.

Para facilitar a análise da associação da insegurança alimentar com a RDPC, e sua evolução no tempo, vamos definir as seguintes variáveis binárias (com valor 0 e 1$)$ :

IA1 - assume valor 1 para pessoas de domicilios com qualquer grau de insegurança alimentar

IA2 - assume valor 1 para pessoas de domicílios com insegurança alimentar moderada ou grave

IA3 - assume valor 1 para pessoas de domicilios com insegurança alimentar grave

SA1 - assume valor 1 para pessoas de domicílios com segurança alimentar; é o complemento de IA1, isto é, $\mathrm{SA} 1=1-\mathrm{IA} 1$

SA2 - assume valor 1 para pessoas de domicílios com segurança alimentar ou com insegurança leve; é o complemento de IA2, isto é, SA2 = $1-$ IA2
SA3 - assume valor 1 para pessoas de domicilios sem segurança alimentar grave; é o complemento de IA3, isto é, SA3 = $1-$ IA3

As Figuras 5, 6 e 7 permitem observar como a insegurança alimentar está associada ao nível de RDPC e como, entre 2004 e 2013, ela caiu em todos os décimos da distribuição da RDPC. A Figura 8 mostra as curvas de Lorenz e as curvas de concentração de IA1, IA2, IA3 e SA1 em 2004 e 2013. 
Figura 5. Proporção de pessoas com insegurança alimentar de qualquer grau (IA1 $=1)$ em cada décimo da distribuição da RDPC

2004

2013
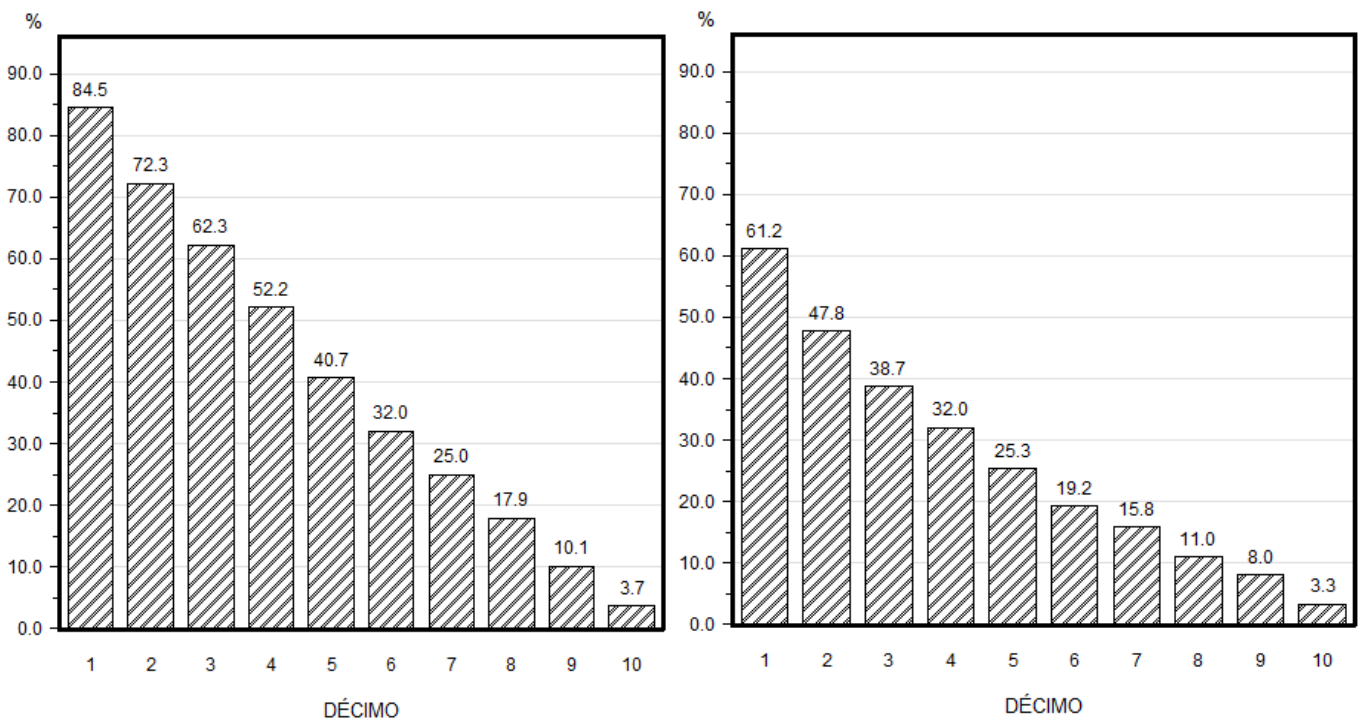

Figura 6. Proporção de pessoas com insegurança alimentar moderada ou grave $(\mathrm{IA} 2=1)$ em cada décimo da distribuição da RDPC

2004

2013
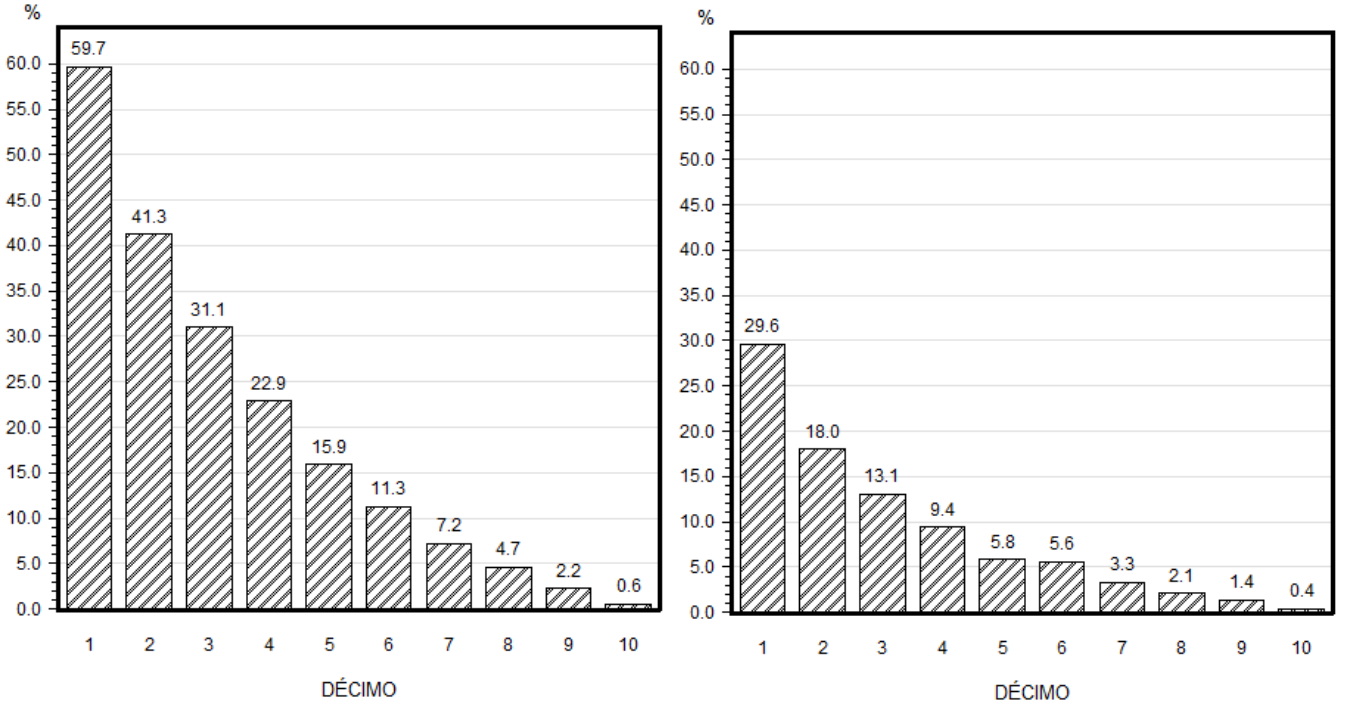
Figura 7. Proporção de pessoas com insegurança alimentar grave $(\mathrm{IA} 3=1)$ em cada décimo da distribuição da RDPC

2004

2013
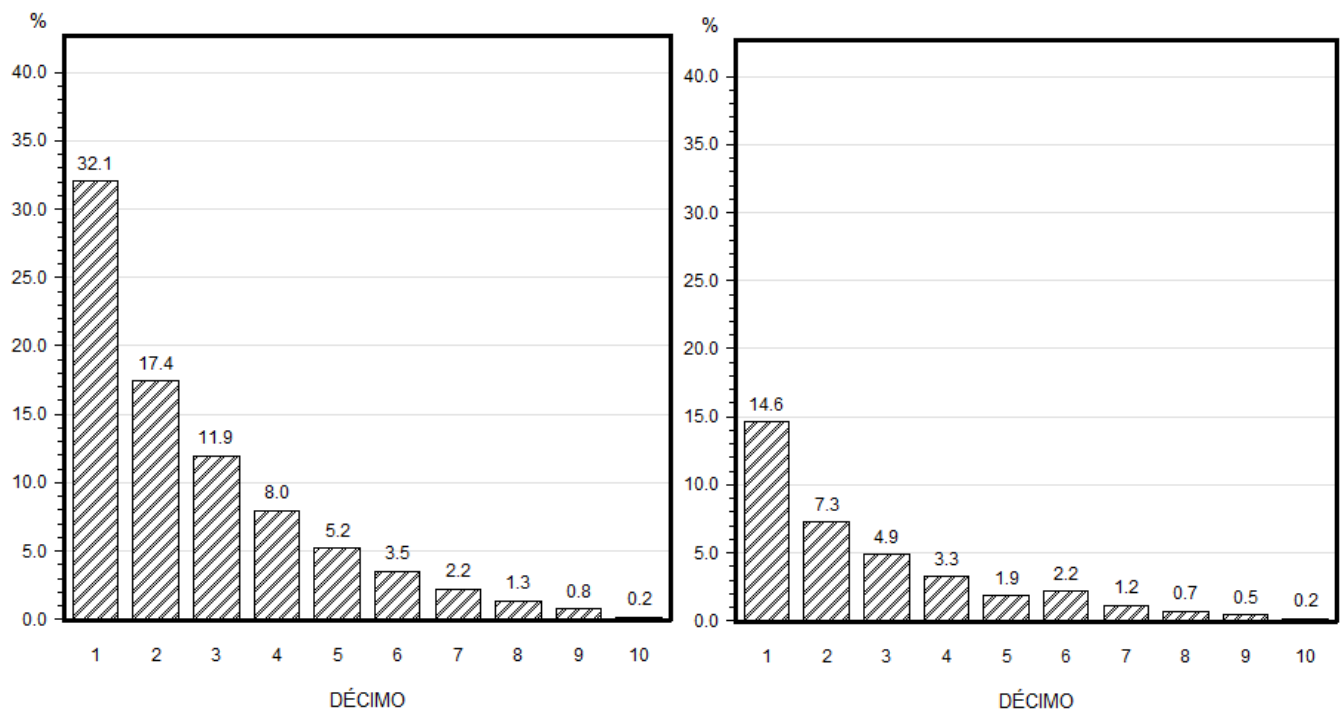

Figura 8. Curva de Lorenz da distribuição da RDPC e as curvas de concentração para IA1, IA2, IA3 e SA1

2004

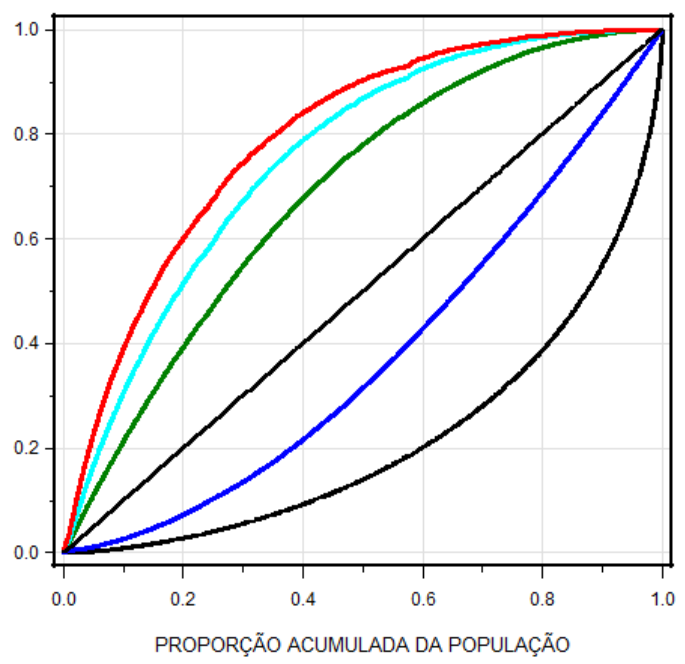

$\underset{\text { IA3 }}{\longrightarrow}$ LORENZ $_{\text {SA1 }}=$
2013

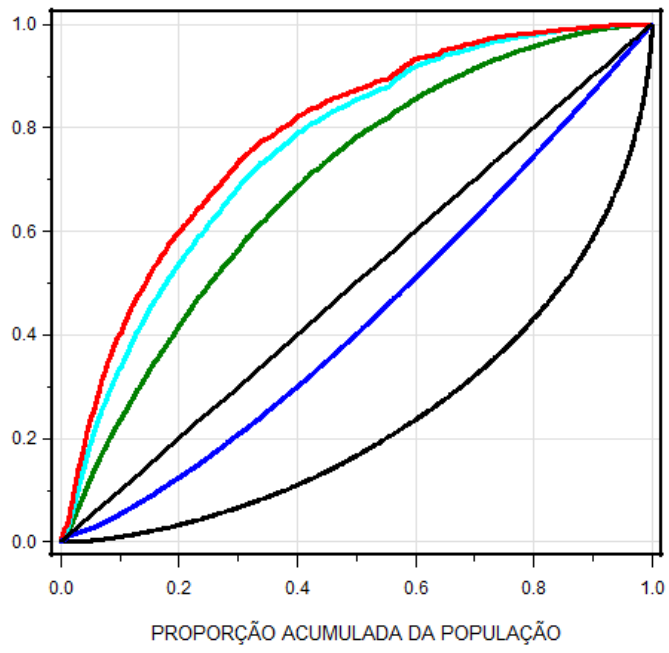

IA2 
Observa-se, na Figura 8, que IA3 é mais concentrada nos pobres do que IA2 e esta, por sua vez, é mais concentrada do que IA1. Isso é confirmado pelos valores das razões de concentração apresentadas na Tabela 2.

Tabela 2. Médias $(\boldsymbol{\mu})$ e razões de concentração $(C)$ para insegurança alimentar (IA1), insegurança alimentar moderada ou grave (IA2), insegurança alimentar grave (IA3) e para os respectivos complementos: segurança alimentar (SA1), ausência de insegurança moderada ou grave (SA2) e ausência de insegurança grave (SA3), sempre em relação à RDPC. Brasil: 2004, 2009 e 2013

\begin{tabular}{c|c|c|c|c|c|c}
\hline \multirow{2}{*}{ Variável } & \multicolumn{2}{|c|}{2004} & \multicolumn{2}{c|}{2009} & \multicolumn{2}{c}{2013} \\
\cline { 2 - 7 } & $\boldsymbol{\mu}$ & $\boldsymbol{C}$ & $\boldsymbol{\mu}$ & $\boldsymbol{C}$ & $\boldsymbol{\mu}$ & $\boldsymbol{C}$ \\
\hline IA1 & 0,401 & $-0,372$ & 0,345 & $-0,330$ & 0,262 & $-0,382$ \\
IA2 & 0,197 & $-0,508$ & 0,134 & $-0,505$ & 0,089 & $-0,513$ \\
IA3 & 0,083 & $-0,583$ & 0,059 & $-0,572$ & 0,036 & $-0,566$ \\
SA1 & 0,599 & 0,248 & 0,655 & 0,173 & 0,738 & 0,136 \\
SA2 & 0,803 & 0,124 & 0,866 & 0,078 & 0,911 & 0,050 \\
SA3 & 0,917 & 0,053 & 0,941 & 0,036 & 0,964 & 0,021 \\
RDPC1 & 619,3 & 0,570 & 793,2 & 0,540 & 938,7 & 0,525 \\
\hline
\end{tabular}

Fonte: Elaborada a partir dos microdados da PNAD (IBGE)

${ }^{1}$ Média em R\$ de setembro-outubro de 2013 e índice de Gini da RDPC

Note-se que em 2013 a curva de concentração de segurança alimentar (SA1) está mais próxima do bissetor do primeiro quadrante do que em 2004. Como mostra a Tabela 2, a respectiva razão de concentração cai de 0,248 em 2004 para 0,136 em 2013. As razões de concentração de SA2 e SA3 também diminuem no período, da mesma maneira que o índice de Gini.

Considerando o conjunto das razões de concentração para ISA1, ISA2 e ISA3, não se observa uma tendência de redução do seu valor absoluto. A insegurança alimentar permanece concentrada nos relativamente pobres.

Por outro lado, observa-se clara tendência decrescente, de 2004 a 2013, nas razões de concentração de SA1, SA2 e SA3: a segurança alimentar está cada vez menos concentrada nos ricos.
A expressão (17) mostra que a razão de concentração de uma variável complementar de uma binária com valores 0 e 1 é uma função matemática da média e da razão de concentração da variável original. Isso significa que os dados apresentados nas linhas para SA1, SA2 e SA3 na Tabela 2 já estão implícitos nos dados apresentados nas três primeiras linhas do corpo da Tabela. Se a razão de concentração de IA1 se mantém estável e sua média cai, necessariamente a razão de concentração de SA1 cai.

\section{Razões de concentração da insegurança alimentar nas áreas urbanas e rurais}

A Figura 9 mostra as curvas de Lorenz da RDPC e as curvas de concentração de IA1, IA2, IA3 e SA1 para pessoas em domicilios urbanos e para pessoas em domicilios rurais. 
Figura 9. Curvas de concentração de IA1, IA2, IA3 e SA1 em relação à RDPC. Brasil, 2013, urbano e rural

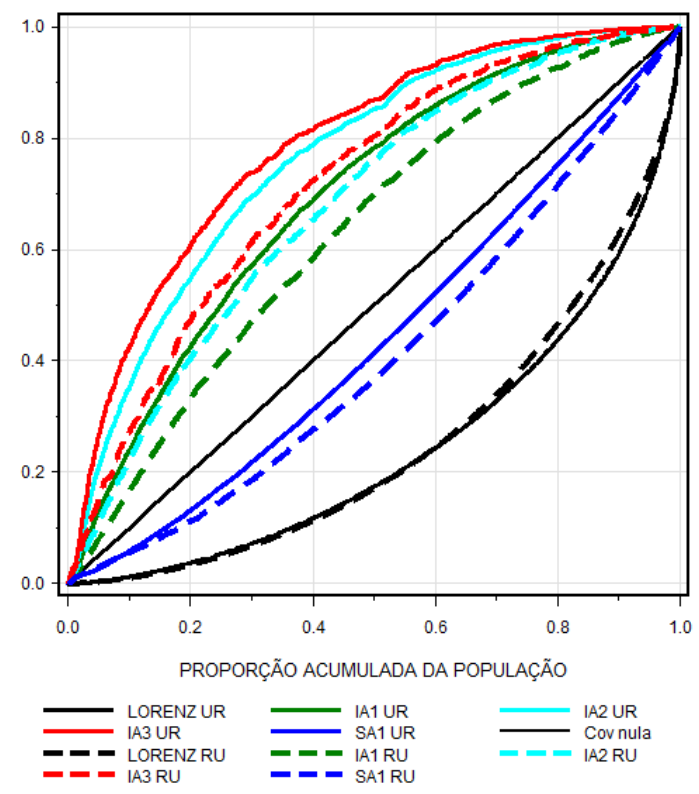

Observa-se que a curva de Lorenz para a área urbana delimita uma área de desigualdade um pouco maior do que a curva de Lorenz para a área rural.
Consequentemente, o índice de Gini para a área urbana $(0,515)$ é maior do que o da área rural $(0,501)$, como mostra a Tabela 3.

Tabela 3. Razões de concentração de insegurança alimentar (IA1), insegurança alimentar moderada ou grave (IA2) e insegurança grave (IA3) e respectivos complementos — segurança alimentar (SA1), ausência de insegurança moderada ou grave (SA2) e ausência de insegurança grave (SA3) —, sempre em relação à RDPC. Brasil, 2004, 2009 e 2013, conforme situação do domicílio

\begin{tabular}{c|c|c|c|c|c|c}
\hline \multirow{2}{*}{ Variável } & \multicolumn{2}{|c|}{2004} & \multicolumn{2}{c|}{2009} & \multicolumn{2}{c}{2013} \\
\cline { 2 - 7 } & Urbana & Rural & Urbana & Rural & Urbana & Rural \\
\hline IA1 & $-0,389$ & $-0,289$ & $-0,342$ & $-0,279$ & $-0,389$ & $-0,265$ \\
IA2 & $-0,532$ & $-0,384$ & $-0,524$ & $-0,406$ & $-0,526$ & $-0,359$ \\
IA3 & $-0,610$ & $-0,457$ & $-0,596$ & $-0,444$ & $-0,577$ & $-0,435$ \\
SA1 & 0,238 & 0,288 & 0,171 & 0,187 & 0,120 & 0,178 \\
SA2 & 0,117 & 0,146 & 0,074 & 0,093 & 0,043 & 0,068 \\
SA3 & 0,050 & 0,061 & 0,034 & 0,042 & 0,019 & 0,029 \\
RDPC1 & 0,559 & 0,517 & 0,531 & 0,489 & 0,515 & 0,501
\end{tabular}

Fonte: Elaborado a partir dos microdados da PNAD

${ }^{1}$ Índice de Gini da RDPC 
Tanto a Figura 9 como a Tabela 3 mostram que a concentração das variáveis para insegurança alimentar (IA1, IA2 e IA3) em relação à RDPC é substancialmente maior na área urbana do que na área rural. Isso está associado à maior disseminação da insegurança alimentar nas áreas rurais, com maior proporção de domicílios pobres, mas também se deve, pelo menos em parte, ao fato de muitos pobres rurais terem parte de suas necessidades alimentares atendidas por meio de produção para autoconsumo. É importante ter em mente que a medida de renda da PNAD não capta o valor da produção para autoconsumo. Se fosse utilizada uma medida de renda que incluísse o valor da produção para autoconsumo, certamente a diferença entre razões de concentração da insegurança alimentar rurais e urbanas seria menor.

\section{Diferenças regionais na concentração da insegurança alimentar em relação à $\mathrm{RDPC}$}

Observa-se, na Tabela 4, que há um nítido contraste entre o Norte-Nordeste e o Centro-Sul do País no que se refere à segurança alimentar. Em 2013, no Norte e no Nordeste a proporção de pessoas com segurança alimentar fica abaixo de $60 \%$, ao passo que nas demais regiões fica acima de $79 \%$. A proporção de pessoas com insegurança alimentar grave fica abaixo de $2,5 \%$ no Sudeste, no Sul e no Centro-Oeste, mas é igual a ou superior a $6 \%$ no Norte e no Nordeste. Esse contraste regional é claramente associado ao nível da RDPC média, que fica abaixo de $\mathrm{R} \$ 630,00$ no Norte e no Nordeste e supera os $\mathrm{R} \$ 1.000,00$ nas demais regiões 9 .

Examinando as razões de concentração apresentados na Tabela 5 , verifica-se que a concentração da insegurança alimentar nos relativamente pobres de cada região é mais intensa nas regiões com maior RDPC média. $\mathrm{O}$ valor absoluto da razão de concentração da insegurança alimentar em qualquer nível (IA1) supera 0,35 no Sudeste, no Sul e no Centro-Oeste, e fica abaixo de 0,25 no Norte e no Nordeste.
Verifica-se, também, que a razão de concentração para segurança alimentar (SA1) supera 0,16 nas duas regiões mais pobres e fica abaixo de 0,10 nas demais regiões. O grau de concentração da segurança alimentar em favor dos ricos da região é maior nas duas regiões mais pobres.

9 A importância da RDPC como um dos determinantes da segurança alimentar é analisada em Hoffmann, 2014 [13]. 
Tabela 4. Pessoas em domicílios particulares permanentes com informação sobre RDPC e segurança alimentar, RDPC média e porcentagens de pessoas em cada categoria de segurança alimentar em 6 regiões do Brasil em 2013

\begin{tabular}{c|r|r|r|r|r|r}
\hline \multirow{2}{*}{ Estatística } & \multicolumn{7}{c}{ Região } \\
\cline { 2 - 7 } & Norte & Nordeste & MG+ES+RJ & SP & \multicolumn{1}{c}{ Sul } & C. Oeste \\
\hline População (1.000 pessoas) & 16.369 & 53.515 & 38.713 & 39.736 & 27.325 & 14.195 \\
RDPC média (R\$) & 627,7 & 599,4 & $1.023,4$ & $1.218,0$ & $1.149,8$ & $1.157,3$ \\
\% com IA1 & 41,0 & 42,4 & 19,9 & 13,2 & 16,6 & 20,3 \\
\% com IA2 & 16,7 & 15,9 & 5,3 & 3,9 & 4,5 & 5,6 \\
\% com IA3 & 7,8 & 6,0 & 2,0 & 1,9 & 2,0 & 2,4 \\
\% com SA1 & 59,0 & 57,6 & 80,1 & 86,8 & 83,4 & 79,7 \\
\% com SA2 & 83,3 & 84,1 & 94,7 & 96,1 & 95,5 & 94,4 \\
\% com SA3 & 92,2 & 94,0 & 98,0 & 98,1 & 98,0 & 97,6 \\
\hline
\end{tabular}

Tabela 5. Razões de concentração de insegurança alimentar (IA1), insegurança alimentar moderada ou grave (IA2) e insegurança grave (IA3) e dos respectivos complementos — segurança alimentar (SA1), ausência de insegurança moderada ou grave (SA2) e ausência de insegurança grave (SA3) —, sempre em relação à RDPC, em seis regiões do Brasil, em 2013

\begin{tabular}{|c|c|c|c|c|c|c|}
\hline \multirow{2}{*}{ Variável } & \multicolumn{6}{|c|}{ Região } \\
\hline & Norte & Nordeste & $\mathrm{MG}+\mathrm{ES}+\mathrm{RJ}$ & SP & Sul & C. Oeste \\
\hline IA1 & $-0,238$ & $-0,243$ & $-0,366$ & $-0,398$ & $-0,404$ & $-0,358$ \\
\hline IA 2 & $-0,384$ & $-0,357$ & $-0,516$ & $-0,523$ & $-0,522$ & $-0,486$ \\
\hline IA3 & $-0,461$ & $-0,435$ & $-0,565$ & $-0,557$ & $-0,604$ & $-0,579$ \\
\hline SA1 & 0,165 & 0,179 & 0,091 & 0,061 & 0,081 & 0,091 \\
\hline SA2 & 0,077 & 0,067 & 0,029 & 0,021 & 0,025 & 0,029 \\
\hline SA3 & 0,039 & 0,028 & 0,012 & 0,011 & 0,012 & 0,014 \\
\hline $\mathrm{RDPC}^{1}$ & 0,517 & 0,536 & 0,508 & 0,489 & 0,462 & 0,525 \\
\hline
\end{tabular}

Fonte: Elaborado a partir dos microdados da PNAD de 2013

${ }^{1}$ Índice de Gini da RDPC

\section{À guisa de conclusão} do artigo.

Segue uma lista de tópicos abordados ao longo

Não se deve confundir a razão de concentração (ou índice de concentração) com uma medida da desigualdade de uma distribuição. A razão de concentração está diretamente associada à covariância entre duas variáveis. Trata-se de avaliar a concentração de uma variável $Y$ em relação a uma outra variável $X$.

A “correção" de Erreygers está conceitualmente errada e deve ser esquecida.

A insegurança alimentar no Brasil diminuiu substancialmente de 2004 a 2013, mas não há tendência de redução do valor absoluto da razão de concentração 
da insegurança alimentar em relação à distribuição da RDPC.

Como a RDPC é menor nas áreas rurais do País, a insegurança alimentar é maior nessas áreas. Mas o valor absoluto da razão de concentração da insegurança alimentar em relação à RDPC é maior nas áreas urbanas do que nas áreas rurais.

Embora a insegurança alimentar seja mais intensa nas regiões Norte e Nordeste, é nas regiões do Centro-Sul do Brasil que ela está mais concentrada nos domicílios relativamente pobres de cada região.

\section{REFERÊNCIAS}

[1] O’Donnel O., Doorslaer E., Wagstaff A.; Lindelow M. Analyzing health equity using household survey data: a guide to techniques and their implementation. Washington: The World Bank, 2008. 220 p.

[2] Kakwani N., Wagstaff A., Doorslaer E. Socioeconomic inequalities in health: measurement, computation and statistical inference. Journal of Econometrics 1997; 77(1):87103, 1997.

[3] Monteiro CA et al. Desigualdades socioeconômicas na baixa estatura infantil: a experiência brasileira, 1974-2007. Estudos Avançados, 2013; 27(78):35-49.

[4] Wagstaff A. The bounds of the concentration index when the variable of interest is binary, with an application to immunization inequality. J. Health Econ. 2005; 14 (4):42932, 2005.
[5] Wagstaff A. On the measument of inequalities in health. Soc. Sci. Med. 1991;33(5):545-57.

[6] Hoffmann R. Transferências de renda e desigualdade no Brasil (1995-2011). In: Campello T., Neri MC. (org.) Programa Bolsa Família: uma década de inclusão e cidadania. Brasília: IPEA, 2013, p. 207-216.

[7] Erreygers G. Correcting the concentration index. J. Health Econ. 2009; 28(2):504-15.

[8] Wagstaff A. Correcting the concentration index: a comment. J. Health Econ. 2009; 28 (2):516-20.

[9] Erreygers G. Correcting the concentration index: a reply to Wagstaff. J. Health Econ. 2009; 28(2):521-4.

[10] Instituto Brasileiro de Geografia e Estatística IBGE. Pesquisa Nacional por Amostra de Domicílios: segurança alimentar 2004. Rio de Janeiro: IBGE, 2006. $127 \mathrm{p}$.

[11] Instituto Brasileiro de Geografia e Estatística IBGE. Pesquisa Nacional por Amostra de Domicílios: segurança alimentar 2004/2008. Rio de Janeiro: IBGE, 2010. 171 p.

[12] Instituto Brasileiro de Geografia e Estatística IBGE. Pesquisa Nacional por Amostra de Domicílios: segurança alimentar 2013. Rio de Janeiro: IBGE, 2014. 139p.

[13] Hoffmann R. Brasil, 2013: mais segurança alimentar. Segur Aliment Nutr. 2014; 21(2):422-436. 


\section{Apêndice A. Evolução das razões de concentração da posse de alguns bens de consumo duráveis em relação à renda domiciliar per capita}

Neste apêndice é analisada a evolução da concentração da posse de televisão em cores, geladeira, rádio, máquina de lavar roupa e telefone, sempre em relação à RDPC. Para isso são utilizados os microdados das PNADs de 1995 a 2013, com a última versão dos fatores de ponderação da amostra fornecidos pelo IBGE. Cabe lembrar que a PNAD não foi realizada em 2000 e 2010, quando foram realizados Censos Demográficos. A pergunta, na PNAD, se refere à existência ou não do bem em cada domicílio, mas nossas unidades de análise foram as pessoas. Assim, foi atribuído valor 1 à variável binária para televisão em cores para toda pessoa em cujo domicílio a pergunta sobre a existência desse bem de consumo durável recebeu resposta afirmativa ("sim"). Para "geladeira" considerou-se, indiferentemente, as com 1 ou 2 portas.

No que se refere à posse de telefone, até 1999 foi feita uma única pergunta: "Este domicílio tem telefone?". A partir de 2001 passaram a ser feitas duas perguntas: sobre presença de "telefone móvel celular" e de "telefone fixo convencional". Atribuímos valor 1 à variável binária "telefone" quando existia qualquer um dos dois tipos de telefone.
Como até 2003 a PNAD não coletava dados na área rural da antiga região Norte, para manter a comparabilidade ao longo do tempo, eliminamos da amostra, a partir de 2004, os dados referentes a essa área.

A figura A1 mostra como variou, de 1995 a 2013, a proporção de pessoas em cujos domicílios estava presente cada um dos bens duráveis considerados e a figura A2 mostra a evolução da respectiva razão de concentração.

Figura A1. Proporção de pessoas em domicílios com alguns bens de consumo duráveis. Brasil, 1995 a 2013

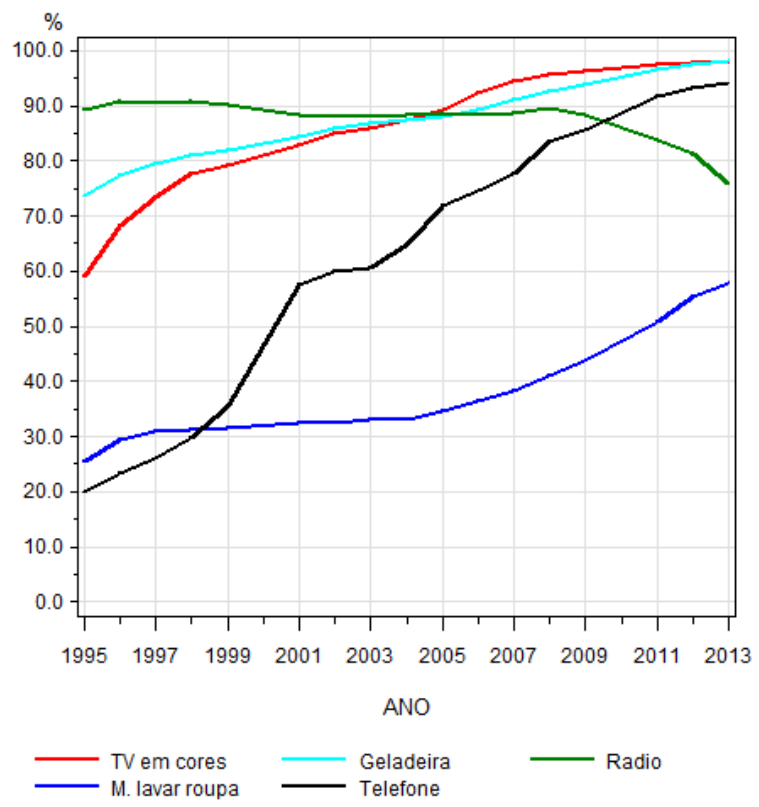


Figura A2. Evolução das razões de concentração da posse de alguns bens de consumo duráveis em relação à RDPC. Brasil, 1995 a 2013

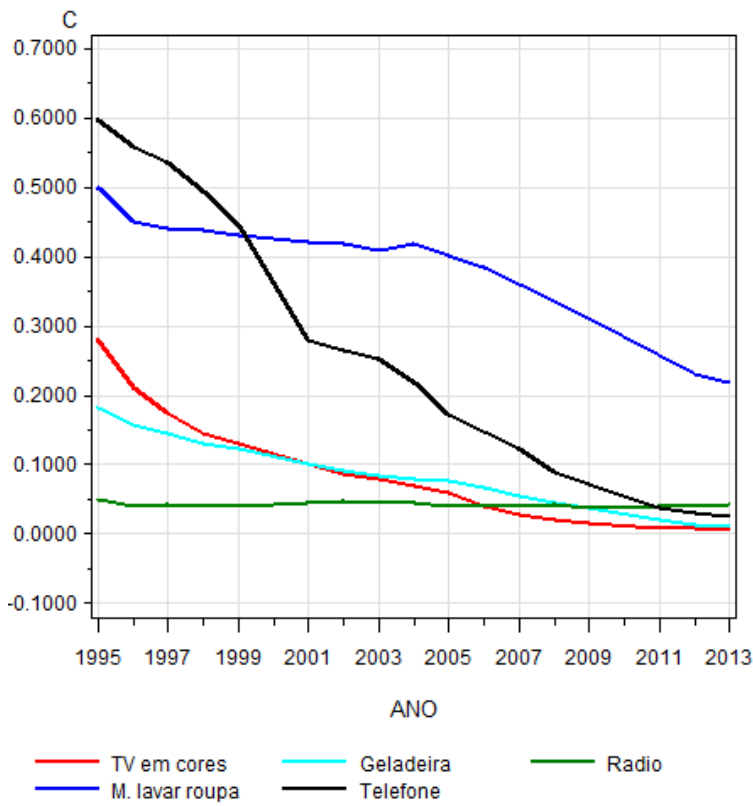

A porcentagem de pessoas cujo domicílio tem televisão em cores cresce de 59,1\% em 1995 para 97,8\% em 2013. A proporção de pessoas cujo domicilio tem geladeira é inicialmente maior (73,6\%), mas chega a nível semelhante em $2013(98,0 \%)$. Para a posse de máquina de lavar roupa a proporção cresce de $25,4 \%$ para $57,7 \%$. A disseminação da posse desses bens duráveis pela maior parte da população é acompanhada pela redução da respectiva razão de concentração em relação à RDPC. No caso da posse de televisão em cores e de geladeira esta chega a quase zero $(0,01)$ em 2013 . Para a posse de máquina de lavar roupa, a razão de concentração em relação à RDPC cai de 0,499 em 1995 para 0,218 em 2013.

O fenômeno impressionante, nesse período de 18 anos, é o crescimento da proporção de pessoas cujo domicilio tem telefone, de 20,1\% para 94,3\%, associado com o progresso tecnológico no setor e a privatização dos serviços (o leilão de privatização da Telebrás ocorreu em julho de 1998). Em 1995 a posse de um telefone ainda era um privilégio de pessoas relativamente ricas, com uma razão de concentração de 0,597 em relação à RDPC. Em 2013 o panorama é muito diferente, com razão de concentração igual a 0,025 .
O rádio era um bem durável já bastante disseminado em 1995, presente no domićlio de 89,2\% das pessoas. Como mostra a figura A1, essa proporção se manteve próxima de $90 \%$ até 2008 e depois mostra tendência decrescente, chegando a 75,8\% em 2013. Mas o número mais reduzido de pessoas com posse do bem não se concentra nos relativamente ricos. $\mathrm{A}$ razão de concentração permanece próxima de 0,04 ao longo de todo o período analisado. 